\title{
Im Osten nichts Neues? Ziele, Inhalte und erste Ergebnisse der Östlichen Partnerschaft
}

\author{
Katrin Böttger*
}

Am 7. Mai 2009 verabschiedeten die 27 EU-Mitgliedstaaten gemeinsam mit den sechs östlichen Nachbarn Ukraine, Moldau, Belarus, Georgien, Armenien und Aserbaidschan in Prag eine Erklärung zur Östlichen Partnerschaft (ÖP). Sie soll einen neuen Abschnitt der Beziehungen, die im Rahmen der Europäischen Nachbarschaftspolitik (ENP) geregelt sind, eröffnen.

Auf den ersten Blick erscheint es überraschend, dass es jetzt zu einer ausdifferenzierten Politik gegenüber den osteuropäischen Staaten gekommen ist, nachdem vergleichbare Initiativen in der Vergangenheit gescheitert waren. Bezüglich der ÖP lässt sich jedoch beobachten, dass die Europäische Union trotz kleiner Machtspielchen im Vorfeld des Prager Gipfels in diesem Bereich der Außenpolitik gegenüber den osteuropäischen Staaten zunehmend als kollektiver Akteur auftritt. Dies gilt insbesondere in Bezug auf Ordnungsvorstellungen und mittelfristige Politikziele. Hingegen bringt die institutionelle Ausgestaltung abgesehen von einer multilateralen Komponente keine überraschenden Neuerungen mit sich. Aufgrund des kollektiven Auftretens konnte die ÖP jedoch zustande kommen. Dieser Umstand wurde zudem durch interne und externe Rahmenbedingungen begünstigt und führte zu einer zügigen Implementation. Auch die Reaktionen auf die ÖP nicht nur durch die EU-Mitgliedstaaten, sondern auch durch die östlichen Partner und Russland zeigen, dass die Europäische Union zunehmend als eigenständiger Akteur wahrgenommen wird.

Im Inneren der Europäischen Union ist hervorzuheben, dass die Mitgliedstaaten zwar langfristig unterschiedliche Vorstellungen bezüglich der osteuropäischen Staaten haben. Insbesondere die mittelosteuropäischen Staaten streben eine EU-Mitgliedschaft für die osteuropäischen Nachbarstaaten an, welche von der Mehrheit der EU-Mitgliedstaaten abgelehnt wird. Entscheidend für die Entstehung der ÖP waren aber die mittelfristig nahe beieinanderliegenden Vorstellungen darüber, wie die osteuropäischen Staaten Schritt für Schritt an die Europäische Union herangeführt werden sollen. Hinzu kommt, dass auch zwischen den einzelnen EU-Institutionen eine große Einigkeit über die Ausgestaltung der ÖP zu beobachten ist.

Unter den externen Rahmenbedingungen, welche die Entstehung der ÖP befördert haben, ist zum einen die „Union für den Mittelmeerraum“1 zu nennen. Die Initiative für die südliche Nachbarschaft wirkte gleichsam als Katalysator und führte bei allen Mitgliedstaaten dazu, dass eine eigenständige Politik für die osteuropäischen Nachbarn ebenfalls akzeptiert wurde. Überraschenderweise hat jedoch auch der russisch-georgische Konflikt im August 2008 den Entstehungsprozess der ÖP eher beschleunigt als gebremst.

Die Implementation der ÖP ist insbesondere durch eine neue multilaterale Komponente gekennzeichnet, die durch das gemeinsame Verständnis aller EU-Mitgliedstaaten vom ho-

* Dr. des. Katrin Böttger, Wissenschaftliche Mitarbeiterin, Institut für Europäische Politik, Berlin; Lehrbeauftragte an der Universität Leipzig. Der Aufsatz ist im Rahmen des IEP-Schwerpunktprogramms „Dialog Europa der Otto Wolff-Stiftung“ im Rahmen des Projekts „Die Beziehungen der Europäischen Union zu den östlichen Nachbarn - zwischen Nachbarschafts- und Erweiterungspolitik“ entstanden. Die Autorin dankt Mariella Falkenhain für wertvolle Anmerkungen und Thomas Schüler für vielfältige Zuarbeit.

1 Offizieller Name „Union für den Mittelmeerraum“, im Folgenden abgekürzt als „Mittelmeerunion“. 
hen Stellenwert der Kooperation möglich wurde. Ein weiterer Beleg für die Relevanz, welche der ÖP beigemessen wird, ist ihre zügige Umsetzung. Auch die ausführlichen und differenzierten Reaktionen auf die Politik zeigen, welche Bedeutung ihr von den Mitgliedstaaten, von allen osteuropäischen Partnerstaaten und auch von Russland zugeschrieben wird. Bevor der Einfluss dieser Rahmenbedingungen auf die Entstehung der ÖP näher erläutert wird, erfolgt eine kurze Einordnung der ÖP in ihren größeren Rahmen, die ENP, die auch nach Einführung der ÖP weiterhin Bestand hat.

\section{Die Europäische Nachbarschaftspolitik}

Die ENP entstand im Zuge der EU-Osterweiterung, als die osteuropäischen Staaten Ukraine, Moldau und Belarus direkte Nachbarn der Europäischen Union wurden. Ihr Ziel war es, Stabilität in Osteuropa zu erreichen und Trennlinien an der neuen EU-Außengrenze zu vermeiden. Der Fokus der Aufmerksamkeit richtete sich noch aus einem weiteren Grund gen Osten: Insbesondere die ukrainische Regierung äußerte seit Ende der 1990er-Jahre ,europäische Ambitionen ‘ bis hin zum Ziel einer EU-Mitgliedschaft. Diese Vorstellungen stießen in der Europäischen Union jedoch auf Widerstand. Deshalb begannen die EU-Mitgliedstaaten, sich über ein gemeinsames Konzept zur Neugestaltung der Beziehungen zu den osteuropäischen Staaten zu verständigen. Diese waren zu dem Zeitpunkt lediglich im Rahmen von Partnerschafts- und Kooperationsabkommen geregelt, die jährliche Gipfeltreffen und regelmäßige Konsultationen auf Minister- und Beamtenebene vorsahen. Die Beziehungen der Europäischen Union zu diesen neuen Nachbarstaaten, aber auch zu den Staaten im südlichen Kaukasus ${ }^{2}$ und jenseits des Mittelmeers ${ }^{3}$ sind aufbauend auf Partnerschafts- und Kooperationsabkommen beziehungsweise Assoziierungsabkommen seit 2004 durch die ENP als Rahmenkonzept geregelt. Alle genannten Staaten verfügen derzeit nicht über eine EU-Beitrittsperspektive. Von der ENP ausgenommen sind Russland und der Westliche Balkan. Während die Beziehungen zu Russland im Rahmen der strategischen Partnerschaft und seit 2003 mit dem Ziel, vier Gemeinsame Räume in den Bereichen Wirtschaft, äußere und innere Sicherheit sowie Bildung einzurichten, geregelt sind, verfügen die Staaten des Westlichen Balkans ${ }^{4}$ über eine Beitrittsperspektive. ${ }^{5}$

$\mathrm{Zu}$ den Zielen der ENP zählen Normenexport und Reformen in Bezug auf Demokratie, Menschenrechte und Rechtsstaatlichkeit sowie Wirtschaftshilfe, technische Hilfe und die Gewährung von Marktzugängen. Die ENP verfolgt gegenüber den Nachbarstaaten einen bilateralen Ansatz. Aufbauend auf Strategiepapieren der Europäischen Kommission sind ihre wichtigsten Instrumente Länderberichte und Aktionspläne, Fortschrittsberichte und das neue Finanzinstrument ENPI. ${ }^{6}$ Ergänzend sind Länderstrategiepapiere und Nationale Indikativprogramme hinzugekommen, die zur Weiterführung älterer Instrumente dienen. Die Länderstrategiepapiere für den Zeitraum 2007-2013 sollen die Reformprogramme des jeweiligen Staates unterstützen und schließen an die Partnerschafts- und Kooperationsabkommen sowie die Aktionspläne an. Die Nationalen Indikativprogramme für den Zeitraum 2007-2010 set-

2 Georgien, Armenien, Aserbaidschan.

3 Marokko, Algerien, Tunesien, Libyen, Ägypten, Israel, Palästinensische Autonomiebehörde, Jordanien, Libanon, Syrien.

4 Kroatien, Bosnien und Herzegowina, ehemalige jugoslawische Republik Mazedonien, Albanien, Montenegro, Serbien, Kosovo gemäß Resolution 1244 des UN-Sicherheitsrates.

5 Europäische Kommission: Mitteilung der Kommission. Europäische Nachbarschaftspolitik. Strategiepapier, KOM (2004) 373.

6 Das Europäische Nachbarschafts- und Partnerschaftsinstrument (ENPI) verfügt im Zeitraum 2007-2013 über eine Summe von 12 Milliarden Euro. 
zen Schwerpunkte fest, welche mithilfe des ENPI finanziert werden sollen. ${ }^{7}$ Diese durch die ENP angewandten Instrumente beinhalten einerseits Reformanreize und Transformationshilfen für die Partnerstaaten. Andererseits sollen sie im Interesse der Europäischen Union Sicherheit und Stabilität exportieren.

\section{Interne Rahmenbedingungen bei der Entstehung der Östlichen Partnerschaft}

Die internen Rahmenbedingungen, welche die Entstehung der ÖP beeinflusst haben, zeigen sich bei der Analyse ihres Entstehungsprozesses. Hervorzuheben ist hierbei die schnelle Einigung auf mittelfristige Ziele und das routinierte Ineinandergreifen der Aktivitäten der EU-Institutionen.

\section{Der polnisch-schwedische Entwurf}

Im Mai 2008 stellten Polen und Schweden beim Ministertreffen erstmals Vorschläge für eine ÖP vor. ${ }^{8}$ Für eine solche Ausdifferenzierung der ENP hatte es bereits seit Ende der 1990er-Jahre zahlreiche Initiativen von polnischer, tschechischer sowie von deutscher Seite gegeben. Hierzu zählen die Eastern Dimension, ${ }^{9}$ die ENPplus ${ }^{10}$ und die Initiative für eine neue Ostpolitik. ${ }^{11}$ Sie alle waren von dem Gedanken getragen, die ENP auszudifferenzieren, da sie sich auf eine große und heterogene Gruppe von europäischen und nicht-europäischen Staaten bezieht. Ein weiterer Grund für eine erneute Initiative Polens und Schwedens im Jahr 2008 war die Bilanz der ENP, die nach ersten Anläufen und der vielversprechenden Orangenen Revolution in der Ukraine im Jahr 2004 von fehlenden Erfolgen gekennzeichnet ist. Diese werden von den mittelosteuropäischen Staaten auf die mangelnde Attraktivität der Politik und ihre Perspektivlosigkeit bezüglich eines Beitritts zurückgeführt, während die Europäische Union Schwächen und zuweilen Widerstände der Partnerstaaten bei der Implementation konstatiert.

Im Gegensatz zu früheren Initiativen einer geografisch eingegrenzten Politik für die östlichen Nachbarstaaten verlief der polnisch-schwedische Vorschlag zu einer ÖP nicht im Sande, ${ }^{12}$ sondern wurde von den Institutionen der Europäischen Union aufgenommen. Polen hebt als Ziel dieser Initiative hervor, in der Nachbarschaftspolitik nach der Einführung der Mittelmeerunion wieder zu einer Balance zwischen Süd und Ost zu kommen. ${ }^{13}$ Dies findet die Zustimmung der anderen Mitgliedstaaten, unter anderem auch Deutschlands, dem an ei-

7 Die drei Hauptschwerpunkte für die Ukraine sind beispielsweise: Unterstützung der demokratischen Entwicklung und des verantwortlichen Regierens, Unterstützung der Reformen im Bereich der Regulierung und Aufbau von Verwaltungskapazitäten und Unterstützung der Infrastrukturentwicklung. Vgl. Europäische Kommission: Europäisches Nachbarschafts- und Partnerschaftsinstrument. Ukraine. Nationales Richtprogramm 2007-2010, 2007.

8 Polish-Swedish proposal. Eastern Partnership, Mai 2008, abrufbar unter: http://www.tepsa.eu/docs/ draft_proposal_eastern_partnership.pdf (letzter Zugriff: 04.08.2009); Rat der Europäischen Union: Mitteilung an die Presse. 2870. Tagung des Rates: Allgemeine Angelegenheiten und Außenbeziehungen am 26.-27. Mai 2008, Dok. 9868/08 (Presse 141).

9 Ministry of Foreign Affairs of the Republic of Poland: non-paper, 2003; Česká Republika: Wider Europe NonPaper, 2003.

10 Rosa Balfour/Antonio Missiroli: Reassessing the European Neighbourhood Policy, European Policy Centre: EPC Issue Paper Nr. 54, Juni 2007.

11 Iris Kempe: Eine neue Ostpolitik. Europas Antwort auf die Regenbogenrevolutionen, in: Osteuropa 9/2005, S. 21-34. Vgl. ausführlich: Katrin Böttger: Die Entstehung der europäischen Nachbarschaftspolitik. Eine Untersuchung der Rolle individueller und kollektiver Akteure, Dissertation, Tübingen im Erscheinen.

12 Dies war z.B. bei der ENPplus der Fall, da der Entwurf zu viel Widerspruch von Seiten Spaniens und anderer südlicher EU-Mitgliedstaaten erhielt. Vgl. Katinka Barysch: What to expect from the German presidency, Centre for European Reform: briefing note, Januar 2007.

13 Agence France Presse: Polen stößt Osteuropa-Partnerschaft an, 23.05.2008. 
ner ausgewogenen Politik gegenüber den Nachbarn in Ost und Süd gelegen ist. Dass die Planungen zur ÖP alle EU-Mitgliedstaaten ansprechen sollen, folgt aus der schon 2003 von Polen in seinem Non-Paper geäußerten Ansicht, dass die Chancen und Probleme der östlichen Nachbarn die Europäische Union als Ganzes betreffen. ${ }^{14}$

\section{Weiterentwicklung durch die EU-Institutionen}

Der Europäische Rat im Juni 2008 begrüßte die Weiterentwicklung der östlichen Dimension in bilateralen und multilateralen Strukturen. Er betonte die Differenzierung zwischen drei Ebenen der Zusammenarbeit, welche im Entwurf zur ÖP angelegt aber noch nicht systematisch herausgearbeitet sind. Zum Ersten nennt er die bilaterale Kooperation zwischen der Europäischen Union und den einzelnen Partnerstaaten sowie zwischen den Partnerstaaten untereinander, zum Zweiten die regionale Kooperation zwischen der Europäischen Union und der Region als Ganzes und zum Dritten die ENP als umfassendes Rahmenkonzept. ${ }^{15}$ Neue Aspekte der Politik sind in diesem Zusammenhang die Unterscheidung der Ebenen, insbesondere die Betonung der Kooperation der osteuropäischen Staaten untereinander, sowie die Schaffung multilateraler Strukturen. Der Europäische Rat forderte die Kommission auf, bis zum Rat im Frühjahr 2009 Vorschläge für eine ÖP zu entwickeln. Jedoch ersuchte er sie auf der außerordentlichen Tagung am 1. September 2008 anlässlich des russisch-georgischen Konflikts, diese Vorschläge schon im Dezember des gleichen Jahres vorzulegen, um einen Beschluss bereits im Frühjahr 2009 zu ermöglichen. ${ }^{16}$

Insgesamt deckt sich der Kommissionsentwurf in weiten Teilen mit den polnisch-schwedischen Vorschlägen. Jedoch ergänzt er sie an vielen Stellen durch Hebel politischer Konditionalität. So übernahm die Kommission die Aufteilung in bilaterale und multilaterale Komponenten der Zusammenarbeit. Dies gilt auch für die Möglichkeit, mit den Nachbarländern neue Assoziierungsabkommen (sogenannte ,Enhanced Agreements') abzuschließen, deren Ziel eine stärkere politische Anbindung ist. Als Voraussetzung für ihre Verhandlung nennt der Entwurf jedoch eine Vielzahl politischer Benchmarks, die vor Beginn solcher Verhandlungen erfüllt sein müssen. Die Abkommen sollen durch engeren Bezug zum EU-Recht und den EU-Standards zu mehr Konvergenz führen und auch die Zusammenarbeit in der Gemeinsamen Außen- und Sicherheitspolitik und der Europäischen Sicherheits- und Verteidigungspolitik fördern. ${ }^{17}$ Die Kommission betont jedoch, dass die ÖP keine Beitrittsperspektive beinhalte.

Einige Vorschläge fasst der Entwurf unter griffigen Titeln zusammen: Er spricht hinsichtlich der zukünftigen Visapolitik, die im Gegensatz zum polnisch-schwedischen Entwurf zunächst Erleichterungen der Visumvergabe und nur langfristig Visafreiheit vorsieht, von „Mobilitäts- und Sicherheitspakten“. Diese beinhalten auch gemeinsame Visumstellen. Des Weiteren umfassen sie auch Aufgaben für die EU-Seite. Hierbei handelt es sich insbesondere um Unterstützungsmaßnahmen für die Grenzverwaltung, den Datenschutz und die Kriminalitätsbekämpfung. In Bezug auf den Freihandel der Nachbarstaaten untereinander

14 Ministry of Foreign Affairs of the Republic of Poland: non-paper, 2003. Dies bekräftigt auch die polnische EU-Kommissarin Danuta Hübner. Vgl. Philippa Runner: Poland and Sweden to pitch 'Eastern Partnership' idea, in: EUobserver.com, 22.05.2008.

15 Rat der Europäischen Union: Tagung des Europäischen Rates vom 19./20. Juni 2008 in Brüssel. Schlussfolgerungen des Vorsitzes, Dok. 11018/1/08.

16 Ebenda, S. 19; Rat der Europäischen Union: Außerordentliche Tagung des Europäischen Rates vom 1. September 2008 in Brüssel. Schlussfolgerungen des Vorsitzes, Dok. 12594/2/08.

17 Europäische Kommission: Mitteilung der Kommission an das Europäische Parlament und den Rat. Östliche Partnerschaft, KOM (2008) 823, S. 4. 
spricht der Entwurf von einer „Neighbourhood Economic Community“, die sich am europäischen Wirtschaftsraum orientieren könnte. Um weitreichende und umfassende Handelszonen zu errichten, sollen langfristig alle tarifären und viele nichttarifäre Handelshemmnisse abgebaut werden. Dies bedeutet eine Harmonisierung bei Standards und Zertifizierung und somit eine schrittweise Rechtsangleichung an das EU-Recht. Beim Handel der Nachbarstaaten mit der Europäischen Union schlägt die Kommission zunächst sektorspezifische Maßnahmen vor. Für eine weitreichende und umfassende Freihandelszone zwischen der Europäischen Union und den Nachbarstaaten, welche eines der Ziele der Assoziierungsabkommen ist, gilt die Mitgliedschaft in der Welthandelsorganisation (WTO) als Voraussetzung. ${ }^{18}$ Ziel ist es, die Anziehungskraft des europäischen Binnenmarktes zu nutzen. ${ }^{19}$ Hinsichtlich der Freihandelszonen formuliert die Kommission zurückhaltender als der polnisch-schwedische Entwurf.

Die EU-Außenminister einigten sich im Februar 2009 auf die Einführung der ÖP, ${ }^{20}$ beim Europäischen Rat im März 2009 bildete die ÖP einen Schwerpunkt. In seinen Schlussfolgerungen begrüßt er die Einrichtung der ÖP, betont das verstärkte Engagement der Europäischen Union in den und für die östlichen Partnerstaaten. Des Weiteren werden noch einmal die Prinzipien der Zusammenarbeit hervorgehoben, die auch für die ENP gelten, namentlich gemeinsame Verantwortung, Differenzierung und Konditionalität. Der Europäische Rat hebt zudem wie schon der polnisch-schwedische Entwurf die Bedeutung hervor, welche die Entwicklung der Beziehungen der Staaten untereinander für die Region hat. Neben Assoziierungsabkommen nennt der Europäische Rat die von der Kommission erarbeiteten Vorschläge umfassender Freihandelszonen, Visum- und Rückübernahmeabkommen sowie das Ziel der Energieversorgungssicherheit als Instrumente zur Umsetzung. Er sieht für die ÖP folgende Strukturen vor: alle zwei Jahre Treffen auf Ebene der Staats- und Regierungschefs der ÖP und jährliche Treffen der Außenminister. Hinzu kommen vier thematische Plattformen zu den Kernbereichen der Zusammenarbeit: Demokratie, wirtschaftliche Integration, Energie und direkte Kontakte zwischen den Menschen. Ziel war es, bis zum Gipfeltreffen im Mai in Konsultationen mit den Partnerländern eine gemeinsame Erklärung zu erarbeiten. ${ }^{21}$ Die schnelle Entwicklung der ÖP wurde durch den weitgehenden Konsens zwischen den einzelnen EU-Institutionen und unter den EU-Mitgliedstaaten befördert. Ein Zeichen hierfür ist der Umstand, dass der Europäische Rat kaum hinter den Empfehlungen der Kommission zurückblieb. Zudem wurde die Einigung auf eine formelle Erklärung nie infrage gestellt, da kein Mitglied mit einem Veto drohte.

Auch unmittelbar vor dem Gipfel in Prag gab es nur einige wenige Uneinigkeiten über die Abschlusserklärung, von denen einige auf sprachlicher Ebene ausgetragen wurden. Im Wesentlichen entspricht die Abschlusserklärung jedoch dem Ratsbeschluss von März 2009 und den Empfehlungen der Kommission aus dem Dezember 2008. Unklar war, ob die sechs Staaten als ,Europäische Staaten“ betitelt werden sollten. Dies entsprach insbesondere den Vorstellungen der neuen Mitgliedstaaten Polen, Tschechien, Slowakei und Ungarn, welche eine weitreichende Erklärung vorzogen. Eine andere Gruppe von Staaten, unter anderem

18 Diese ist bei der Ukraine, Moldau, Georgien und Armenien gegeben, lediglich Belarus und Aserbaidschan erfüllen diese Voraussetzung noch nicht. Vgl. World Trade Organization: Accessions, abrufbar unter: http:// www.wto.org/english/theWTO_e/acc_e/acc_e.htm (letzter Zugriff: 12.08.2009).

19 Benita Ferrero-Waldner: Ambitious New Partnership for the East, Rede vor dem polnischen Parlament am 27.11.2008, SPEECH/08/672, S. 3.

20 Frankfurter Allgemeine Zeitung: EU einig über Ost-Partnerschaft, 24.02.2009.

21 Rat der Europäischen Union: Tagung des Europäischen Rates in Brüssel vom 19./20. März 2009. Schlussfolgerungen des Vorsitzes, Dok. 7880/1/09. 
Deutschland, Frankreich, Großbritannien, Italien und die Beneluxstaaten wünschten sich eine zurückhaltendere Formulierung, die in keinem Fall eine Beitrittsperspektive ausdrücken sollte. Sie bevorzugten eine Formulierung wie sie die Erklärung des Europäischen Rates wählte, die von „Partnerländern“ spricht. ${ }^{22}$ Man einigte sich schließlich nicht auf ,Östliche Partner", wie es die Kommission vorschlug, sondern auf „Osteuropäische Partner“, die in der Folge des Dokuments lediglich „Partnerländer“" genannt werden. ${ }^{23}$

Hinsichtlich einer möglichen Visafreiheit für die Bürger der osteuropäischen Staaten herrschte unmittelbar vor dem Gipfel noch Unklarheit. Ursprünglich hatte die Kommission in ihrer Mitteilung vom 3. Dezember 2008 eine vollständige Visafreiheit vorgeschlagen. Dies hatte auch die tschechische Ratspräsidentschaft in einen ersten Entwurf übernommen. Hierfür spricht, dass Reiseerleichterungen das EU-Image verbessern. ${ }^{24}$ Dies ging jedoch einigen Mitgliedstaaten, unter anderem Deutschland, zu weit. ${ }^{25}$ Ein Alternativvorschlag sah einen „graduellen Prozess“ vor, sodass der Gipfel sich schließlich darauf einigte, ,schrittweise auf die vollständige Abschaffung der Visumpflicht als langfristiges Ziel“" hinzuarbeiten. ${ }^{26}$ In Bezug auf die wirtschaftliche Kooperation ist nun nicht mehr explizit die Rede von einer Wirtschaftsgemeinschaft, wie es noch im Kommissionsvorschlag der Fall war, sondern lediglich von einem Netz weitreichender Freihandelszonen.

Am 7. Mai 2009 fand in Prag der Gründungsgipfel zur ÖP statt, auf dem die gemeinsame Erklärung unterzeichnet wurde. ${ }^{27}$ Sie erhielt zwar die Unterstützung aller EU-Mitgliedstaaten, jedoch gingen dem Gipfel zahlreiche Absagen verschiedener Staats- und Regierungschefs voraus, die andere Termine vorschützten und nicht am Gipfel teilnahmen. Nicht anwesend waren der britische Premierminister Gordon Brown, Spaniens Ministerpräsident José Luis Rodríguez Zapatero und der italienische Ministerpräsident Silvio Berlusconi. ${ }^{28}$ Auch Nicolas Sarkozy nahm nicht teil und ließ nur durch seinen Premierminister erklären, welche hohe Bedeutung Frankreich dem Projekt beimesse. Während beim Gipfel zur Mittelmeerunion am 13. Juli 2008 bis auf den tschechischen Ministerpräsidenten Mirek Topolánek praktisch alle Staats- und Regierungschefs nach Paris gekommen waren, wurden nur etwas mehr als die Hälfte der EU-27 in Prag durch ihre Staats- oder Regierungschefs repräsentiert. ${ }^{29}$

Der Umstand, dass viele EU-Mitgliedstaaten lediglich von ihren Außenministern oder Staatssekretären vertreten worden waren, lässt einige Autoren schlussfolgern, dass der Gipfel in Prag im Besonderen und die ÖP im Allgemeinen ein zum Scheitern verurteilter Misserfolg sind. ${ }^{30}$ Andererseits stand die Tatsache, dass nur wenige Staats- und Regierungschefs anwesend waren, der Erarbeitung konkreter Ergebnisse in der Folgezeit nicht im Wege. Dies

22 Rat der Europäischen Union: Tagung des Europäischen Rates 19./20. März, 2009, Anlage 2; Agence Europe: EU/Eastern Partnership: Final negotiations over language in joint " $27+6$ " declaration ahead of Prague summit, 05.05.2009.

23 Rat der Europäischen Union: Gemeinsame Erklärung des Prager Gipfeltreffens zur Östlichen Partnerschaft. Prag, 7. Mai 2009, Dok. 8435/09 (Presse 78).

24 Tomas Valasek: What the economic crisis means for the EU's eastern policy, Centre for European Reform: policy brief, April 2009, S. 2.

25 Ebenda, S. 3.

26 Rat der Europäischen Union: Tagung des Europäischen Rates 19./20. März, 2009, Anlage 2; Agence Europe: EU/Eastern Partnership: Final negotiations over language, 2009; Rat der Europäischen Union: Gemeinsame Erklärung des Prager Gipfeltreffens, 2009, S. 7.

27 Ebenda.

28 Andrew Rettman: Big names to stay away from Prague summit, in: EUobserver.com, 04.05.2009.

29 EUbusiness.com: EU holds landmark summit with ex-Soviet states, 07.05.2009.

30 Wiebke Drescher: The Eastern Partnership and Ukraine. New Label - Old Products?, Zentrum für Europäische Integrationsforschung: Discussion Paper C194/2009; Nicu Popescu: A Swedish-led listening tour of the East, abrufbar unter: http://blogs.euobserver.com/popescu/2009/06/22/a-swedish-listening-tour-of-the-east/ (letzter Zugriff: 12.08.2009). 
zeigt sich daran, dass sich auf Arbeitsebene in den Plattformen schon weiterführend mit diesen Ergebnissen befasst wird. Insgesamt haben die internen Faktoren somit die Einführung einer kollektiven Politik gegenüber den osteuropäischen Staaten eher befördert.

\section{Der Einfluss externer Faktoren bei der Entstehung der Östlichen Partnerschaft}

Auch einige externe Faktoren haben sich positiv auf die Entstehung der ÖP ausgewirkt. Es ist wenig überraschend, dass nach Einführung der Mittelmeerunion wiederum nach geografischer Balance gestrebt wird. Hingegen erstaunt der Umstand, dass der russisch-georgische Konflikt im August 2008 nicht wie bei früheren Spannungen zwischen Russland und seinen Nachbarn zu Zurückhaltung oder Auseinanderdriften der EU-Mitgliedstaaten geführt hat, sondern diese vielmehr in ihrer Politik bestärkte und diese sogar beschleunigte.

\section{Austarieren zwischen Ost und Süd}

Ein wichtiger Grund für den Erfolg des polnisch-schwedischen Entwurfs ist der Umstand, dass die EU-Mittelmeeranrainer - nachdem die Mittelmeerunion ins Leben gerufen worden war - dazu bereit waren, ebenfalls eine eigene Politik für den osteuropäischen Raum zu akzeptieren, zumal diese anders als die Mittelmeerunion keine eigenen Institutionen vorsieht. Diesen Zusammenhang stellte auch der Rat für Außenbeziehungen her, indem er auf die Aussprache des Europäischen Rates zur Mittelmeerunion im März 2008 verwies. ${ }^{31}$

Bei der ÖP handelt es sich somit um eine weitere in einer langen Reihe von Pendelbewegungen bei denen die Europäische Union sich immer abwechselnd schwerpunktmäßig mit den östlichen Regionen und den südlichen Anrainern befasst hat; sie reichen von der Mittelmeerpolitik der Europäischen Gemeinschaft in den 1980er-Jahren über die EU-Osterweiterung, den Barcelona-Prozess, die ENP, die Mittelmeerunion bis hin zur ÖP.

Während die südlichen EU-Mitgliedstaaten - allen voran Frankreich - in der Vergangenheit jede Politik abgelehnt hatten, die sich speziell den osteuropäischen Staaten widmet, lieBen sie sich unter den veränderten politischen Bedingungen darauf ein. Zwar sind sie nicht bereit, von dem Finanzschlüssel abzurücken, der zwei Drittel der ENPI-Gelder für die südlichen Nachbarn und ein Drittel für die östlichen Nachbarn vorsieht. ${ }^{32}$ Dennoch sieht Sarkozy die Notwendigkeit ein, eine ÖP ins Leben zu rufen, insbesondere angesichts des Kaukasuskonflikts und der drohenden Lieferschwierigkeiten im Energiebereich. Diese hätten unmittelbare Auswirkungen auf die Sicherheit und Stabilität der Europäischen Union gehabt. Deshalb müsse eine aktivere Politik zunächst in dieser Region angestrebt werden. ${ }^{33}$ Dieses Zugeständnis weist auf eine Veränderung der französischen Einstellung hin. Bis zu diesem Zeitpunkt hatte Frankreich zumeist kein Verständnis für die Relevanz der osteuropäischen Staaten für die Stabilität und Sicherheit der Europäischen Union gezeigt, sondern sich ganz auf Stabilitäts- und Sicherheitsprobleme im Mittelmeerraum konzentriert.

\section{Der russisch-georgische Konflikt}

Die tatsächliche Einführung einer ÖP wurde somit maßgeblich durch die Ereignisse im russisch-georgischen Konflikt im August 2008 beschleunigt. Diese brachten die europä-

31 Rat der Europäischen Union: Mitteilung an die Presse. 2870. Tagung des Rates, 2008, S. 24.

32 Valentina Pop: EU ministers at odds over energy and eastern projects, in: EUoberver.com, 24.02.2009; Valentina Pop: EU expanding its 'sphere of influence', in: EUobserver.com, 21.03.2009.

33 Hubert Gehring/Tomislav Delinic/Marcel Preising/Zbyněk Králík/Katharina Hierl: Tschechische EU-Ratspräsidentschaft. Lissabon, Klaus und die Finanzkrise: Europa hat einige Hürden zu bewältigen, Konrad-Adenauer-Stiftung: 3. Newsletter zur tschechischen EU-Ratspräsidentschaft, Februar 2009. 
ischen Mitgliedstaaten und die Kommission zu der Überzeugung, dass Stabilität in der Region nur durch eine engere Anbindung an die Europäische Union erreicht werden kann. ${ }^{34}$ Anstatt einem Konflikt mit Russland aus dem Wege zu gehen, um Risiken zu vermeiden, engagiert sich die Europäische Union noch stärker in der Region, die von Russland als seine exklusive Einflusssphäre angesehen wird. Aus polnischer Sicht bestätigte der Konflikt zudem die wiederholt geäußerten Befürchtungen, dass Russland zu alten, sowjetischen Vorgehensweisen zurückkehren könnte. ${ }^{35}$

Zusätzliche Faktoren, die sich beschleunigend auf die weitere Entwicklung der ÖP auswirkten, waren die entstehende Finanz- und Wirtschaftskrise und der immer wieder aufflackernde Gasstreit zwischen Russland und der Ukraine, ${ }^{36}$ der im Jahr 2009 durch veränderte Zahlungsbedingungen und ein neues Preisregime zu monatlichen anstatt jährlichen Finanzierungsengpässen führte. ${ }^{37}$ Die Gaskrise im Winter 2008/09 ist auch für Benita FerreroWaldner ein weiterer Beleg für die dringende Notwendigkeit für eine ÖP. ${ }^{38}$

\section{Die Implementation der Östlichen Partnerschaft}

Die Implementation der ÖP begann nur wenige Wochen nach dem Beschluss zu ihrer Einführung mit den Treffen der multilateralen Plattformen und der Initiierung eines zivilgesellschaftlichen Forums. Somit lässt sich auch nach dem Beschluss zur ÖP beobachten, dass der Konsens über ihre Notwendigkeit über Lippenbekenntnisse hinausgeht, da EU-Institutionen und Mitgliedstaaten sich weiterhin aktiv mit ihr befassen. In diesem Zusammenhang bildet die multilaterale Kooperation einen neuen Schwerpunkt. Eine Beschleunigung und neue Schwerpunktsetzung lässt sich auch bei der Finanzierung der ÖP beobachten.

\section{Multilaterale Plattformen und zivilgesellschaftliches Forum}

Bereits im Juni beziehungsweise Juli 2009 kamen die multilateralen Plattformen zu den Themen Demokratie, wirtschaftliche Integration, Energie und direkte Kontakte zwischen den Menschen zusammen. Teilnehmer der Plattformen sind Vertreter aller beteiligten Staaten, das heißt aller 27 EU-Mitgliedstaaten und der sechs Nachbarstaaten, sowie der EU-Institutionen Rat, Europäisches Parlament, Ausschuss der Regionen, Europäischer Wirtschafts- und Sozialausschuss unter Vorsitz der Kommission. ${ }^{39}$ Die Treffen finden auf der Ebene der Mitarbeiter der Ständigen Vertretungen und Außenministerien bis hin zur Botschafterebene (im Falle Aserbaidschans) statt. Innerhalb der einzelnen Plattformen sollen Panel genannte Arbeitsgruppen eingerichtet werden, welche sich mit konkreten Projekten befassen und dem Erfahrungsaustausch dienen. ${ }^{40}$

34 Michael Emerson: Closing down on 2008, Centre for European Policy Studies: CEPS European Neighbourhood Watch Nr. 44, Dezember 2008, S. 1.

35 Radoslav Sikorski: Zeit für eine neue Ostpolitik, in: Der Tagesspiegel, 01.09.2008.

36 Cathrin Kahlweit: Europas Hinterhof, in: Süddeutsche Zeitung, 06.05.2009.

37 Thomas Wiede: Gasrechnung lastet schwer auf der Ukraine, in: Handelsblatt.com, 29.07.2009; Michael Ludwig/Konrad Schuller: Russland und Ukraine unterzeichnen Gasvertrag, in: Frankfurter Allgemeine Zeitung, 19.01.2009.

38 Benita Ferrero-Waldner: Eastern Partnership - an ambitious project for 21st century European foreign policy, 20.02.2009, abrufbar unter: http://ec.europa.eu/external_relations/eastern/docs/eastern_partnership_article_bf w_en.pdf (letzter Zugriff: 19.08.2009); Valentina Pop: EU ministers at odds, 2009.

39 Vgl. Europäische Kommission: Die Östliche Partnerschaft: Beginn der konkreten Umsetzung, Pressemitteilung, IP/09/872, 04.06.2009.

40 Turkish Weekly: Negotiations Between EU and Eastern Partnership Were Equal Dialogue: Azerbaijan's Ambassador to Belgium, 05.06.2009. 
Der Gipfel von Prag übernimmt den Vorschlag der Kommission, dass diese die Treffen der Plattformen, an denen jeweils über 100 Personen teilnehmen, vorbereitet und ihnen vorsitzt. Für die Entscheidung, den Vorsitz dieser Plattformen der Kommission zu übergeben, spricht deren Erfahrung im Umgang mit den Partnerstaaten. Sie ist aber auch ein Beleg für die wachsende Bedeutung und den Einfluss der Kommission in der ENP. So einigten sich die Teilnehmer der Plattformen auf insgesamt sechs Vorreiterinitiativen:

1. Integriertes Grenzverwaltungsprogramm;

2. Fazilität für kleine und mittlere Unternehmen zur Förderung des Privatsektors;

3. Förderung regionaler Strommärkte, der Energieeffizienz und erneuerbarer Energieträger;

4. Entwicklung des südlichen Energiekorridors;

5. Zusammenarbeit bei der Katastrophenvorsorge und der Bewältigung natürlicher und von Menschen verursachter Katastrophen;

6. Initiative zur Unterstützung von Good Governance im Bereich Umweltschutz. ${ }^{41}$

Diese Initiativen sollen die multilaterale Zusammenarbeit sichtbar machen und in den Mittelpunkt rücken. Somit setzt sich die aus der ENP bekannte Arbeitsteilung fort: Während die Mitgliedstaaten Policy-Initiativen entwickeln, übernimmt die Kommission im Rahmen der Implementierung Gestaltungsfunktion.

Ein großer Vorteil der regelmäßigen Treffen in den Plattformen auf Arbeitsebene ist der Umstand, dass hier Annäherungs- und Lernprozesse gestartet werden können. Die Tatsache, dass alle EU-Mitgliedstaaten an den multilateralen Plattformen beteiligt sind, birgt Vor- und Nachteile. Nachteilig ist, dass die Sitzungen sehr groß sind und aus fast 100 Teilnehmern aus den Delegationen bestehen. Fraglich ist, wie in einer so großen Gruppe Erfahrungen zu ,best practices‘ ausgetauscht werden sollen. Von Vorteil ist andererseits, dass diese Länder nicht nur mit den EU-Institutionen sondern mit Vertretern aller Mitgliedstaaten in Kontakt kommen. Zudem bieten die regelmäßigen Plattformtreffen auch die Gelegenheit zu bilateralen Annäherungen am Rande. Dies ermöglicht infolge der kommunikativen Prozesse Wandel durch transnationale Sozialisation. ${ }^{42}$ Insofern können diese Plattformen als ein Schritt in die Richtung einer Antwort auf die Frage gewertet werden, wie es gelingen kann, die Partnerstaaten in europäische Strukturen einzubinden, ohne sie zu vollwertigen Mitgliedern zu machen. Zudem können die Plattformen möglicherweise das alte Ziel der Kommission unterstützen, die Konkurrenz zwischen den einzelnen Partnerstaaten wieder anzufachen, um Reformen zu beschleunigen, wie es schon mit der Einführung der ENP geplant war. Insgesamt könnten sich die informellen Möglichkeiten der Zusammenarbeit aufgrund von Spillover-Effekten als erfolgreicher herausstellen, als die formal vorgegebenen institutionellen Rahmenbedingungen der Plattformen.

Zentrales Thema der Plattform „Demokratie, gute Regierungsführung und Stabilität“ ist die Stärkung der Zivilgesellschaft. Zu diesem Zweck wird zudem ein zivilgesellschaftliches Forum geschaffen, um in diesem Rahmen die Kontakte untereinander und mit den Behörden zu pflegen. Nach einem Konsultationsprozess im Februar und März 2009, der die zivilgesellschaftlichen Organisationen in den Gestaltungsprozess dieses Forums einbezog, legte die Kommission ein Konzeptpapier vor, das auf die Rolle der Zivilgesellschaft im Rahmen der Plattformen, im Rahmen der Schwarzmeersynergie und im Rahmen des zivilgesellschaftli-

41 Vgl. Europäische Kommission: The Eastern Partnership - Flagship Initiatives, abrufbar unter: http://ec.europa.eu/external_relations/eastern/initiatives/index_en.htm (letzter Zugriff: 10.09.2009).

42 Henning Boekle/Volker Rittberger/Wolfgang Wagner: Normen und Außenpolitik: Konstruktivistische Außenpolitiktheorie, Tübinger Arbeitspapiere zur Internationalen Politik und Friedensforschung Nr. 3, Tübingen 1999; Frank Schimmelfennig: Internationale Sozialisation neuer Staaten. Heuristische Überlegungen zu einem Forschungsdesiderat, in: Zeitschrift für Internationale Beziehungen 2/1994, S. 335-355. 
chen Forums eingeht. Das Papier sieht im Wesentlichen die Verbesserung der Kommunikation untereinander und mit den EU-Institutionen vor. Hinzu kommt die Möglichkeit, inhaltlich zu den Plattformen und den Ministertreffen beizutragen. Diese Möglichkeit ist jedoch weder verbindlich noch in anderer Weise konkret benannt. ${ }^{43}$ Ab Ende Juni 2009 erhielten alle potenziell interessierten Gruppen, das heißt Graswurzelorganisationen, Gewerkschaften, Arbeitgeberverbände, NGOs etc. die Möglichkeit, ihr Interesse an einer Teilnahme am zivilgesellschaftlichen Forum zu bekunden. Ein erstes Zusammentreffen des Forums ist für Mitte November 2009 in Brüssel geplant. ${ }^{44}$ Somit bleibt abzuwarten, welchen Einfluss dieses Forum auf die weitere Gestaltung der ÖP haben wird. Es lässt sich jedoch bereits zu diesem Zeitpunkt konstatieren, dass die Kommission - ähnlich wie sie dies bei der ENP getan hat die zivilgesellschaftlichen Gruppen erst nach Abschluss der Politikformulierung in den Politikprozess einbezieht. Bislang wurde dies zum Beispiel in Bezug auf die ENP und das ENPI von den zivilgesellschaftlichen Akteuren wiederholt bemängelt. Des Weiteren forderten sie in der Vergangenheit, die politischen anstelle der wirtschaftlichen Ziele der ENP stärker zu betonen. ${ }^{45}$

Auch die anderen drei Plattformen haben im Juni 2009 das erste Mal getagt und sich mit den Vorreiterinitiativen befasst.

\section{Die finanzielle Ausstattung der Östlichen Partnerschaft}

Die Aussagen der Kommission zur finanziellen Ausstattung der ÖP sind vage und erscheinen widersprüchlich. Die finanzielle Ausstattung der neuen Maßnahmen im Rahmen der ÖP soll von 2010-2013 insgesamt 600 Millionen Euro betragen, von denen 350 Millionen Euro zusätzlich bereitgestellt werden und sich 250 Millionen aus der Umverteilung von ENPI-Mitteln ergeben sollen. ${ }^{46}$ Jedoch weisen die Vertreter einer Gruppe von Ländern unter Führung Frankreichs darauf hin, dass trotz der Aufstockung der Mittel die bei der Annahme der Finanziellen Vorausschau 2007-2013 vereinbarte ,Zwei Drittel-Ein Drittel-Regel ‘ einzuhalten sei. Begründet wird diese vorab erfolgende Aufteilung mit der Kontinuität der Beziehungen zu den Mittelmeeranrainern. Die Einhaltung der Regel würde bedeuten, dass auch die Mittel für den Mittelmeerraum noch einmal aufgestockt werden müssten. ${ }^{47}$ Aus diesem Grund bleibt unklar, woher die zusätzlichen 350 Millionen Euro letztlich stammen werden. ${ }^{48}$

Um über die Finanzmittel möglichst schnell zu verfügen, wird die Zwischenevaluation des Regionalprogramms für die östlichen Partnerstaaten vorgezogen, sodass ein neues Indi-

43 Association for International Affairs: Eastern Partnership: Towards Civil Society Forum. Policy Recommendations for Eastern Partnership Summit, formuliert auf einer Konferenz im Rahmen der tschechischen Ratspräsidentschaft am 05./06.05.2009 in Prag.

44 Europäische Kommission: Eastern Partnership - Civil Society Forum, abrufbar unter: http://ec.europa.eu/ external_relations/eastern/civil_society/index_en.htm (letzter Zugriff: 12.08.2009).

45 Mahmoud Abu Rahma u.a.: European Commission ENP conference, $3^{\text {rd }}$ September. Reflections from Civil Society representatives, Brief und Zusammenfassung der Konferenz adressiert an EU-Kommissionspräsident Barroso und EU-Kommissarin Ferrero-Waldner, 08.10.2007; EurActiv.com: NGOs ask for greater say in EU 'neighbourhood' policy, 27.03.2007.

46 Europäische Kommission: Östliche Partnerschaft, 2008, S. 17-18; vgl. Valasek: What the economic crisis means, 2009, S. 2.

47 Agence Europe: EU/Eastern Partnership: Difficult debate on funding begins - Involvement of third countries to be decided on case by case basis, 25.02.2009.

48 Agence Europe: EU/Eastern Partnership: European Council approves new $€ 600$ million policy towards eastern neighbours, 21.03.2009. 
kativprogramm für 2010-2013 vor Ende des Jahres 2009 beschlossen werden kann. ${ }^{49}$ Ursprünglich war ein neues Indikativprogramm für die Jahre 2011-2013 vorgesehen, das erst im Laufe des Jahres 2010 beschlossen werden sollte..$^{50}$

\section{Reaktionen aus der EU, den östlichen Partnerstaaten und Russland}

Die im Folgenden dargestellten Reaktionen weisen daraufhin, dass die Europäische Union als kollektiver Akteur in Osteuropa wahrgenommen wird. Dies ist jedoch insbesondere im Fall Russlands nicht gleichbedeutend mit einer Akzeptanz dieses Akteurs.

\section{Die Reaktionen der EU-Mitgliedstaaten und der Kommission}

Die Initiative zu einer ÖP stieß bei den Vertretern Deutschlands, Großbritanniens und der Niederlande von Beginn an auf Zustimmung. Die Reaktion der Kommission war hingegen zunächst zurückhaltend. Die für die Außenbeziehungen und die ENP zuständige Kommissarin Benita Ferrero-Waldner betonte, dass es keinen Unterschied zwischen den östlichen und den südlichen Nachbarn gebe und dass ein Mehrwert der neuen Initiative deutlich werden müsse. ${ }^{51}$ Sie ging davon aus, dass die Initiative diesen Mehrwert in den Bereichen Verkehr, Energie, Klimaschutz, Migration und der Bekämpfung des Menschenhandels auf regionaler Ebene erbringen könnte. Sie wies zudem auf Überschneidungen mit der gerade lancierten Schwarzmeersynergie hin, die mit Ausnahme Belarus' aber inklusive Russlands und der Türkei dieselben Staaten umfasst wie die polnisch-schwedische Initiative. ${ }^{52}$

\section{Die Reaktionen aus den östlichen Partnerstaaten}

Im Januar 2009 signalisierte der ukrainische Präsident Viktor Juschtschenko Bereitschaft, die Angebote der Europäischen Union zu nutzen. ${ }^{53}$ Jedoch betonte der ukrainische Außenminister Boris Tarasyuk, dass die ÖP keine Alternative zum Beitritt darstellt, welchen die Ukraine nach wie vor anstrebt. ${ }^{54}$ Auch Georgien und Moldau befürchten, dass es sich bei der ÖP um einen Trostpreis anstelle der gewünschten EU-Mitgliedschaft handelt.

Aus diesem Grund wurde von Seiten der östlichen Partnerstaaten wiederholt Kritik laut. Insbesondere die Ukraine beklagt, dass die ÖP nicht die von manchen Staaten erhoffte Beitrittsperspektive enthält. Diese Frage bleibt aufgrund ihrer Hervorhebung durch den polnischen Außenminister Radosław Sikorski, der sie bei der Vorstellung des Projekts im Mai 2008 betonte, auf der Tagesordnung. ${ }^{55}$ Armenien und Belarus sehen die ÖP hingegen als Chance, der regionalen Isolation zu entkommen. ${ }^{56}$

Ursprünglich sollte die Einbeziehung Belarus' aufgrund fehlender Bemühungen des Landes um Demokratisierung, Achtung der Menschenrechte und Rechtsstaatlichkeit nur auf

49 Europäische Kommission: Concept Note. Priority areas for ENPI Regional East Programme (SP/NIP) for 2010-13, 2009, S. 2.

50 Europäische Kommission: The Mid Term Review of ENPI Strategy Papers and Indicative Programmes. Information Note for Civil Society Organisations, 2009, S. 2-3.

51 Daniel Saameli: EU debattiert über Ost-Partnerschaft. Aussenkommissarin Ferrero-Waldner skeptisch gegenüber einem Vorstoss Polens und Schwedens, in: St. Galler Tagblatt, 27.05.2008.

52 Der Standard: Nach Mittelmeerunion Überlegungen zu „östlicher Partnerschaft“, 26.05.2008.

53 Stephan Malerius/Nico Lange/Anna Reismann: Bewegung in der östlichen EU-Nachbarschaft: Juschtschenko und Lukaschenko nähern sich an, Konrad-Adenauer-Stiftung: KAS Länderbericht, Februar 2009.

54 Agence Europe: EU/Ukraine: Kiev calls for European aid to rescue its economy - Eastern Partnership is not alternative to joining EU, 26.02.2009.

55 Marcin Łapczyński: The European Union's Eastern Partnership: Chances and Perspectives, in: Caucasian Review of International Affairs 2/2009, S. 143-155.

56 Tahir Taghizade: The European Union's Eastern Partnership: What Does It Offer Azerbaijan, in: ADA Biweekly 13/2009, S. 3-5. 
technischer und Expertenebene und nicht auf politischer, ministerieller Ebene erfolgen. ${ }^{57}$ Auch die Kommission betonte, dass eine Beteiligung von der Gesamtentwicklung der EUBelarus-Beziehungen abhinge. ${ }^{58}$ Die Frage der Einbeziehung Belarus' stieß in der Europäischen Union auf eine geteilte Meinung. Abhängig von der Annahme, dass Kooperation oder eher Isolation Demokratisierungstendenzen fördert, sprachen sich die Vertreter der EU-Mitgliedstaaten für oder gegen eine Einladung Belarus' aus. So lehnten sowohl die Vertreter der Niederlande ${ }^{59}$ als auch die Europaabgeordneten mehrheitlich eine Einladung Alexander Lukaschenkas ab, während Italien eine solche befürwortete. ${ }^{60}$ Auch die Vertreter der belarussischen Zivilgesellschaft unterstützten eine Einladung Lukaschenkas. Sie warnten jedoch vor der Fähigkeit des Regimes, demokratische Reformen zu simulieren und forderten deshalb zugleich von der Europäischen Union, Lukaschenka nichts zu versprechen. ${ }^{61}$ Zwar hat der ukrainische Präsident Viktor Juschtschenko Belarus im Januar 2009 aufgefordert, an der ÖP teilzunehmen. ${ }^{62}$ Zudem hatte die Europäische Union das Einreiseverbot für Vertreter des Regimes für weitere neun Monate ausgesetzt. Dennoch nahm die Europäische Union zunächst eine abwartende Haltung bezüglich einer Einladung des belarussischen Präsidenten Alexander Lukaschenka zum Gipfel im Mai 2009 ein. Der tschechische EU-Ratspräsident Karel Schwarzenberg warnte Belarus zudem davor, Abchasiens und Südossetiens Unabhängigkeit anzuerkennen, da dies eine Teilnahme an der ÖP „,sehr, sehr schwierig“ machen würde. ${ }^{63}$

Schließlich kam es bezüglich Belarus zu einem Kompromiss. Mitte April 2009 erhielt Lukaschenka eine offizielle Einladung, ${ }^{64}$ wobei schon vorher signalisiert wurde, dass die EU-Vertreter hofften, dass er nicht persönlich erscheinen werde. ${ }^{65}$ So nahmen beim Gipfel in Prag mit dem Außenminister Sergej Martynow und dem stellvertretenden Ministerpräsidenten Wladimir Semaschko erstmals Vertreter Belarus' an einem EU-Gipfel teil und vertraten Lukaschenka. Belarus nimmt zunächst nur am multilateralen, nicht aber am bilateralen Teil der ÖP teil. Es erhielt jedoch das Angebot, zukünftig auch am bilateralen Teil partizipieren zu können. ${ }^{66}$ Hierzu müssten jedoch die seit Mitte der 1990er-Jahren ruhenden Verhandlungen über ein Partnerschafts- und Kooperationsabkommen wieder aufgenommen werden.

Die Reaktionen aus dem Südkaukasus waren insgesamt verhalten positiv. Der Außenminister Armeniens sieht neue Möglichkeiten für die weitere Entwicklung und Vertiefung der Beziehungen zwischen der Europäischen Union und Armenien. ${ }^{67}$ Er benannte als Ziele Armeniens die politische Assoziierung und wirtschaftliche Integration mit der Europäischen Union. ${ }^{68}$ Auch der aserbaidschanische Botschafter in Tschechien, Tahir Taghizade, betonte,

57 Polish-Swedish proposal. Eastern Partnership, 2008.

58 Europäische Kommission: Östliche Partnerschaft, 2008, S. 4.

59 Agence Europe: EU/Eastern Partnership: Towards presence of Belarus, but not of president Loukashenko, at summit, 31.03.2009.

60 Agence Europe: EU/Eastern Partnership, 18.03.2009.

61 EurActiv.com: Prague pressed to invite Belarus dictator to summit, 04.03.2009.

62 Malerius/Lange/Reismann: Bewegung in der östlichen EU-Nachbarschaft, 2009.

63 Agence Europe: EU/Eastern Partnership: Difficult debate on funding begins, 2009.

64 Agence Europe: EU/Belarus: President Lukashenko invited to 7 May Eastern Partnership launch summit in Prague, 18.04.2009.

65 Agence Europe: Towards presence of Belarus, 2009; Frankfurter Allgemeine Zeitung: Lukaschenka kommt nicht nach Prag, 06.05.2009.

66 Agence Europe: EU/Belarus: EU offers close bilateral relations in exchange for "irreversible" democratic reform - Possible financial aid, 29.07.2009.

67 Turkish Weekly: Eastern Partnership Another Opportunity For Development of Armenia-eu Relations, 16.05.2009.

68 Ministry of Foreign Affairs of the Republic of Armenia: Deputy Foreign Minister of Armenia Arman Kirakossian participates in the preparatory meeting of the EU Eastern Partnership Summit, 11.04.2009. 
dass Aserbaidschan sehr an den Möglichkeiten interessiert ist, welche die ÖP bietet, während er zugleich einräumte, dass Baku noch viele Fragen zu ihrer genaueren Bedeutung habe. Jedoch hob Aserbaidschan zugleich hervor, dass es nicht zur Kooperation mit Armenien bereit sei, solange dieses aserbaidschanisches Territorium besetzt hält. Diese Aussage bezieht sich auf den Konflikt um das Gebiet Bergkarabach. Armenien hält diesen mehrheitlich von Armeniern bewohnten Teil Aserbaidschans mit der Berufung auf das Selbstbestimmungsrecht der Völker seit 1994 besetzt, während Aserbaidschan sich auf seinen Anspruch auf territoriale Integrität beruft. ${ }^{69}$

Zudem zeigt eine genaue Betrachtung der Angebote der EU-Seite, dass viele davon für die Ukraine bereits gelten und umgesetzt werden, während andere Partnerstaaten, insbesondere Armenien und Aserbaidschan kein Interesse an einer solch intensiven Zusammenarbeit haben, da sie jedem Versuch einer Transformation ihres inneren Aufbaus gegenüber misstrauisch sind. ${ }^{70}$ Ein Beispiel hierfür ist das Angebot von Freihandelsabkommen. Ein solches wird mit der Ukraine seit dem WTO-Beitritt im Mai 2008 schon im Rahmen des Enhanced Agreement verhandelt.

Des Weiteren erschwert die Heterogenität der Zielländer die Entwicklung und Umsetzung eines einheitlichen Rahmens. Während die Ukraine nach wie vor einen langfristigen Beitritt anstrebt, interessiert sich Aserbaidschan vor allem für die Zusammenarbeit im Bereich Energie, Armenien hingegen in erster Linie für finanzielle Unterstützung. Das Interesse auf Seiten Belarus' ist offiziell vor allem wirtschaftlicher Natur, während in der belarussischen Öffentlichkeit die Bedeutung der Sicherung der Menschenrechte im Rahmen der ÖP hervorgehoben wird. Wie die Ukraine verfolgen auch Georgien und Moldau einen proeuropäischen Kurs. Trotz dieses grundsätzlichen Problems der Heterogenität der Zielländer kommt der ukrainische Wissenschaftler Vitalii Martyniuk zu folgendem Schluss: „,The Eastern Neighbours have to welcome the Initiative, implement proper work in their countries and ask for more. " ${ }^{\text {71 }}$ In eine ähnliche Richtung geht die Einschätzung des ukrainischen Präsidenten Wiktor Juschtschenko, der die ÖP einen ersten Fahrplan für die Beziehungen mit Europa nennt. ${ }^{72}$

\section{Russland und die Östliche Partnerschaft}

Im ursprünglichen Entwurf war die Einbeziehung Russlands auf Projektebene über das ENPI, das auch für Russland gilt, als Möglichkeit genannt worden. Tatsächlich können nach dem Beschluss vom Prager Gipfel Drittstaaten fallweise in Projekte eingebunden werden. Wie bisher in der ENP werden jedoch weder die Frage nach der Finalität der ÖP mit einer möglichen Beitrittsperspektive noch die Frage nach der Rolle Russlands beantwortet. Gleichwohl signalisiert die ÖP Russland deutlich, dass die Europäische Union sich in ihrem Kurs gegenüber den osteuropäischen Staaten nicht beirren lässt und auf russische Einflusssphären keine Rücksicht nimmt. Deshalb ist es nicht verwunderlich, dass die Initiative bei Russlands politischen Akteuren alles andere als auf Zustimmung stößt. ${ }^{73}$ Der russische Außenminister Sergej Lawrow sieht in der ÖP einen Versuch der Europäischen Union, ihre

69 Vgl. Taghizade: The European Union's Eastern Partnership, 2009.

70 Vgl. auch Rosa Balfour/Antonio Missiroli: Dealing with troubled neighbourhoods, European Policy Centre: Commentary, Februar 2009.

71 Vitalii Martyniuk: Eastern Partnership: Implementation and Monitoring, in: Vitalii Martyniuk (Hrsg.): EU's Eastern Partnership: Additional Possibilities for European Integration of Ukraine, Kiew 2009, S. 61-74, hier S. 61.

72 EUbusiness.com: EU holds landmark summit, 2009.

73 Pop: EU expanding its 'sphere of influence', 2009. 
Einflusssphäre auszudehnen und den Zugang zu fossilen Energiequellen zu sichern. Weiterhin kritisiert Lawrow den Umstand, dass die Europäische Union eine Teilnahme Belarus' an der ÖP nur unter der Bedingung ermöglichen will, dass Belarus die Unabhängigkeit Abchasiens und Südossetiens nicht anerkennt. Lawrow stellt die Frage, ob diese Kopplung als Drohung oder Erpressung zu sehen ist und hebt hervor, dass die Europäische Union souveräne Staaten zwingt, ihre Position aufzugeben, damit die bilateralen Beziehungen nicht zurückgefahren werden. ${ }^{74}$

Dies wurde jedoch vom tschechischen Außenminister Karel Schwarzenberg zurückgewiesen. Auch der Kommissionspräsident José Manuel Barroso bejahte zwar, dass die Europäische Union nach Einfluss in der Region strebe, betonte aber, dass Einflusssphären, wie sie im Kalten Krieg existierten, heute nicht mehr gültig wären. Vielmehr hob Barroso die Bedeutung von , soft power' hervor, da es der Europäischen Union darum ginge, ihre Werte zu verbreiten. ${ }^{75}$

In Reaktion auf den Prager Gipfel wurden die Proteste Russlands lauter, auch wenn die Europäische Union noch einmal unterstrich, dass es ihr nicht um eine Einflusszone, sondern um Stabilität an den Grenzen Europas ginge. ${ }^{76}$ Beim EU-Russland-Gipfel am 21./22. Mai 2009 in Chabarowsk beschuldigte der russische Präsident Dmitrij Medwedjew die Europäische Union, neue Trennlinien zu erzeugen. ${ }^{77}$ Der russische Außenminister sprach bezüglich der ÖP von einer Provokation und hob insbesondere hervor, dass nicht der Eindruck entstehen dürfe, die Staaten müssten sich zwischen Russland und der Europäischen Union entscheiden. Jedoch wiegelte der EU-Botschafter Russlands, Wladimir Tschischow, ab und sagte, er habe die Erfahrung gemacht, dass Initiativen aus Brüssel ohne größere Finanzmittel nicht viel Einfluss erlangen würden. ${ }^{78}$ Problematisch ist für Russland vielmehr das Stellen von Bedingungen und die zeitliche Nähe weiterer EU-Aktivitäten: Zum einen ist hier die unmittelbare Verknüpfung der Aufforderung an Belarus, Südossetien und Abchasien nicht als eigenständige Staaten anzuerkennen, wenn es am Prager Gipfel teilnehmen will, zu nennen und zum anderen die Ankündigung einer bilateralen Unterstützung der Sanierung des ukrainischen Gastransitnetzes ohne die Beteiligung des Hauptlieferanten Russland. ${ }^{79}$ Dennoch scheint Moskau prinzipiell zur Teilnahme an konkreten Projekten der ÖP bereit. ${ }^{80}$

\section{Fazit: Im Osten doch etwas Neues}

Die Mehrzahl der bilateralen Instrumente, Methoden und Strategien der ÖP ist aus früheren Entwürfen, der Politik gegenüber dem Westlichen Balkan ${ }^{81}$ und den Beziehungen zur Ukraine bereits bekannt. Aus diesem Grund hat die ÖP einen nachholenden Charakter, indem sie in einer gemeinsamen Erklärung grundsätzliche Instrumente für die osteuropäischen Staaten zusammenfasst.

74 Agence Europe: EU/Eastern Partnership: Russia suspects EU for wanting to develop "spheres of influence" in Eastern Europe, 24.03.2009.

75 EUbusiness.com: EU offers closer ties with ex-Soviet states, 04.12.2008; Philippa Runner: Brussels to project 'soft power' in post-Soviet zone, in: EUobserver.com, 03.12.2008.

76 Frankfurter Allgemeine Zeitung: „Östliche Partnerschaft“ der EU gegründet, 08.05.2009.

77 BBC News: Russia alarmed over new EU pact, 22.05.2009.

78 Wiener Zeitung: Trügerische Gipfel-Harmonie, 23.05.2009; Frankfurter Allgemeine Zeitung: „Östliche Partnerschaft" der EU gegründet, 2009.

79 Frankfurter Allgemeine Zeitung: Moskau sagt Gespräche mit Kiew ab, 25.03.2009.

80 Susan Stewart: Russland und die Östliche Partnerschaft. Harsche Kritik, punktuelles Kooperationsinteresse, Stiftung Wissenschaft und Politik: SWP-Akuell 21, April 2009.

81 Valentina Pop: Balkans model to underpin EU's 'Eastern Partnership', in: EUobserver.com, 18.09.2008. 
Neu ist an der ÖP jedoch, dass sie ähnlich wie die ENP - aber noch über sie hinausweisend - eine tiefere Integration vorsieht, ohne jedoch ausschließlich der Logik der bisherigen Erweiterungsprozesse zu folgen. Indem sie auf multilaterale Foren setzt, ist die Gleichberechtigung der Partner unter Umständen eher herzustellen, als in den bilateralen Verhandlungen. Während die Mehrzahl der Mitgliedstaaten der Europäischen Union, aber auch Armenien und Aserbaidschan die multilaterale Komponente einer Erweiterung vorziehen, hoffen die Initiatoren der ÖP, Polen und Schweden, ebenso wie die Ukraine auf Fortschritte im Rahmen der bilateralen Komponente. Einerseits entfernt sich die ENP durch ihre Ausgestaltung und Ausdifferenzierung, insbesondere durch die multilaterale Komponente der ÖP von der Erweiterungslogik, erhöht sich die Geschwindigkeit der Umsetzung der Policy und spielen praktische Fragen eine zunehmend wichtige Rolle. Andererseits könnte die zunehmende Implementierung von EU-Standards insbesondere durch die Ukraine bei einem möglichen späteren EU-Beitritt hilfreich sein. Die Europäische Union verhält sich ambivalent, um weder die Ukraine noch Russland zu verprellen, nachdem Russland schon bei der Ankündigung der ÖP sehr empfindlich reagiert hatte.

Im Vergleich zu der Mittelmeerunion ist die ÖP viel weniger institutionalisiert. Sie verfügt nicht über ein eigenes Sekretariat, sondern wird von der Kommission gesteuert. Allerdings befindet sich die Mittelmeerunion noch immer im institutionellen Aufbau und seit dem Gaza-Konflikt im Dezember 2008 gibt es zwischen den Beteiligten nur informellen Kontakt. Hingegen hat sich die ÖP vom ersten Entwurf bis zum formellen Beschluss aller beteiligten Staaten in unter einem Jahr schnell entwickelt. Aus diesem Grund kann die Initiative Polens und Schwedens zur ÖP im Gegensatz zur Mittelmeerunion positiver bewertet werden. Sie weist bereits kurze Zeit nach ihrem Entstehen und den ersten Sitzungen der vier Plattformen mit ihren sechs Vorreiterinitiativen Ergebnisse vor. Diese Initiativen wurden von der Kommission lanciert, sodass diese starken Einfluss auf die Ausgestaltung nimmt.

Unabhängig vom Vergleich der ÖP mit der Mittelmeerunion muss die ÖP nun mit der Implementierung weiter voranschreiten. Dies bedeutet weniger Öffentlichkeit, nimmt damit auf Arbeitsebene zu einem gewissen Grad den Druck von der Frage nach einer EU-Mitgliedschaft und kann den Bürgern der Partnerstaaten eher zugute kommen als die politische Diskussion.

Mit der Beschleunigung der Entwicklung einer ÖP hat die Europäische Union gezeigt, dass ihr die Region - auch aus eigenem Interesse an deren Stabilität - am Herzen liegt. Wie stark dies der Fall ist, würde die Nachbarstaaten sehr interessieren. Da sich die Europäische Union jedoch bezüglich der langfristigen Perspektive für Osteuropa bis heute nicht einig ist, werden sie eine solch konkrete Antwort auf absehbare Zeit nicht erhalten. Ihnen bleibt nichts anderes übrig, als durch die schrittweise Umsetzung der Reformen eine inkrementelle Einstellungsveränderung in der Europäischen Union zu erreichen und die Zeit für sich arbeiten zu lassen, währenddessen vorangegangene Erweiterungen besser verdaut werden können.

Insgesamt wird die ÖP unterschiedlich beurteilt. Manche Autoren heben hervor, dass die alte Nachbarschaftspolitik - bestenfalls ein wenig vertieft - fortgesetzt wird. ${ }^{82}$ Andere Autoren gehen davon aus, dass die ÖP durchaus einen Mehrwert zu verzeichnen hat und die ENP langfristig überflüssig werden könnte, da die ÖP bereits umfassend die bilaterale Ebene mit abdeckt. ${ }^{83}$ Dem stehen jedoch die Bemühungen eines Teils der EU-Mitgliedstaaten entge-

82 Drescher: The Eastern Partnership and Ukraine, 2009; Horst Bacia: Schönheitsoperation für östliche Partner, in: Frankfurter Allgemeine Zeitung, 07.05.2009.

83 Christophe Hillion/Alan Mayhew: The Eastern Partnership - something new or window-dressing, Sussex European Institute: SEI Working Paper Nr. 109, Januar 2009, S. 23. 
gen, die osteuropäischen Staaten nicht zu eigenständig zu betrachten, sondern sie durch die gemeinsame Gruppierung mit den Mittelmeeranrainern strukturell von einer Beitrittsperspektive fernzuhalten.

Einige Autoren sehen den fehlenden Umgang mit den schwelenden Konflikten in der Region kritisch. Zwar hat die Eskalation eines dieser Konflikte, namentlich der um Südossetien und Abchasien zu einer beschleunigten Beschlussfassung zur ÖP geführt; Eingang in diese Initiative hat die direkte Konfliktbearbeitung gleichwohl nicht gefunden. ${ }^{84}$ Dies ist bemerkenswert, da sich die Europäische Union in den Jahren 2008 und 2009 im Rahmen anderer Initiativen bezüglich der schwelenden Konflikte aktiv gezeigt hat. Hier sind insbesondere zwei Missionen zu nennen, welche die Europäische Union in Konfliktgebieten durchführt. Dies ist zum Ersten die seit November 2005 existierende European Union Border Assistance Mission an der moldauisch-ukrainischen Grenze. ${ }^{85}$ Als Zweites ist die nach der diplomatischen Intervention Sarkozys als Ratsvorsitzendem im August 2008 unabhängig von der ÖP lancierte European Union Monitoring Mission hinzugekommen, die seit Oktober 2008 die Umsetzung der in der Folge des Konflikts zwischen Russland und Georgien geschlossenen Abkommen zum Truppenabzug überwacht. ${ }^{86}$

Problematisch für die Zusammenarbeit im Allgemeinen und die Überwindung der schwelenden Konflikte ist zudem, dass auch die Beziehungen der in der ÖP zusammengefassten Staaten untereinander getrübt sind. Insbesondere die Beziehungen zwischen Armenien und Aserbaidschan sind durch den Konflikt um das Gebiet Bergkarabach belastet. Es besteht die Gefahr, dass die genannten Konflikte eine weitere Entwicklung der Region behindern. Die ÖP vermeidet es jedoch zur Beilegung dieser Konflikte einen direkten Beitrag zu leisten.

Die ÖP ist kein revolutionärer großer Wurf. Trotz einiger Mängel kann sie aber als Ausdruck der inkrementellen Weiterentwicklung dieser neuen Politik zwischen Erweiterung und klassischer Außenpolitik gesehen werden. Ihre zukünftige Entwicklung ist abhängig von der innenpolitischen Lage in den Partnerstaaten. Zudem stellt sich die Frage, inwieweit die Ratspräsidentschaften sie weiter fördern werden. Dies ist eher von Schweden in der zweiten Jahreshälfte 2009, Ungarn und Polen (2011) zu erwarten, als von Spanien und Belgien (2010). Abzuwarten bleibt auch, inwieweit den politischen Ergebnissen des Gipfels und der Plattformen tatsächlich Implementierungsergebnisse folgen werden.

Die intensive Beschäftigung der Europäischen Union mit der ENP und ihren regionalen Differenzierungen Mittelmeerunion und ÖP macht ihre gestiegene Relevanz deutlich. Sie ist das Mittel, um auch außerhalb der Europäischen Union Prosperität, Sicherheit und Stabilität anzustreben. Es hat sich gezeigt, dass bei den EU-Mitgliedstaaten hinsichtlich der osteuropäischen Staaten ein Koordinierungsreflex existiert, der nicht nur unter günstigen Rahmenbedingungen greift, wie sie im Inneren der Europäischen Union im Entstehungsprozess der ÖP zu beobachten waren. Wie der georgisch-russische Konflikt offenbart, wird zunehmend auch in Krisensituationen auf gemeinsame Instrumente zurückgegriffen.

84 Rat der Europäischen Union: Gemeinsame Erklärung des Prager Gipfeltreffens, 2009; Eckart D. Stratenschulte: Planquadrat Osteuropa. Die Östliche Partnerschaft der Europäischen Union, in: Osteuropa 5/2009, S. 29-43, hier S. 34.

85 Vgl. Katrin Böttger: Die Regionale Dimension der Europäischen Nachbarschaftspolitik in Osteuropa: Ukraine und Moldau, in: Europäisches Zentrum für Föderalismus-Forschung (Hrsg.): Jahrbuch des Föderalismus 2007, Baden-Baden 2008, S. 589-603.

86 EU Monitoring Mission in Georgia: About EUMM, abrufbar unter: http://www.eumm.eu/en/about_eumm (letzter Zugriff: 12.08.2009). 